\title{
Synthetic prostacyclin agonist, ONO1301, enhances endogenous myocardial repair in a hamster model of dilated cardiomyopathy: A promising regenerative therapy for the failing heart
}

\author{
Kazuhiko Ishimaru, MD, Shigeru Miyagawa, MD, PhD, Satsuki Fukushima, MD, PhD, \\ Atsuhiro Saito, PhD, Yoshiki Sakai, PhD, Takayoshi Ueno, MD, PhD, and Yoshiki Sawa, MD, PhD
}

\begin{abstract}
Objectives: Remodeling of the left ventricle (LV) in idiopathic dilated cardiomyopathy (IDCM) is known to be associated with multiple pathologic changes that endogenous factors, such as hepatocyte growth factor (HGF) and vascular endothelial growth factor (VEGF), protect against. Although a clinically relevant delivery method of these factors has not been established, ONO1301, a synthetic prostacyclin agonist, has been shown to upregulate multiple cardioprotective factors, including HGF and VEGF, in vivo. We thus hypothesized that ONO1301 may reverse LV remodeling in the DCM heart.
\end{abstract}

\begin{abstract}
Methods: ONO1301 dose-dependently added to the normal human dermal fibroblasts and human coronary artery smooth muscle cells in vitro, to measure the expression of HGF, VEGF, stromal cell-derived factor (SDF)-1, and granulocyte-colony stimulating factor (G-CSF), assessed by real-time polymerase chain reaction (PCR) and enzyme-linked immunosorbent assay. $\delta$-Sarcoglycan-deficient J2N-k hamsters, which is an established DCM model, were treated by epicardial implantation of an atelocollagen sheet with or without ONO1301 immersion or sham operation.
\end{abstract}

Results: ONO1301 dose-dependently upregulated expression of these 4 factors in vitro. ONO1301 treatment, which induced dominant elevation of ONO1301 levels for 2 weeks, significantly preserved cardiac performance and prolonged survival compared with the other groups. This treatment significantly upregulated expressions of cardioprotective factors and was associated with increased capillaries, attenuated fibrosis, and upregulation of $\alpha$-sarcoglycan in the DCM heart.

Conclusions: ONO1301 atelocollagen-sheet implantation reorganized cytoskeletal proteins, such as $\alpha$-sarcoglycan, increased capillaries, reduced fibrosis, and was associated with upregulated expression of multiple cardioprotective factors, leading to preservation of cardiac performance and prolongation of survival in the $\delta$-sarcoglycan-deficient DCM hamster. (J Thorac Cardiovasc Surg 2013;146:1516-25)

Idiopathic dilated cardiomyopathy (IDCM) is one of the most critical intractable diseases. The etiology and pathology of IDCM have therefore been intensively investigated to explore other treatment options. ${ }^{1}$ Clinical and functional progression of IDCM has been shown to be closely correlated with the histopathology, such as apoptosis of cardiomyocytes, accumulation of fibrotic components, reduction of vascular density, and remodeling of sarcolemmal/

From the Department of Cardiovascular Surgery, Osaka University Graduate School of Medicine, Osaka, Japan.

This study was financially supported by Grants for the Research and Development of the Myocardial Regeneration Medicine Program from the New Energy Industrial Technology Development Organization (NEDO), Japan.

Disclosures: Yoshiki Sakai is the holder of the patent for ONO1301 encapsulated with PLGA microspheres; All other authors have nothing to disclose with regard to commercial support.

Received for publication Nov 14, 2012; revisions received Feb 8, 2013; accepted for publication Feb 14, 2013.

Address for reprints: Yoshiki Sawa, MD, PhD, Department of Cardiovascular Surgery, Osaka University Graduate School of Medicine, 2-2 Yamadaoka, Suita 565-0871, Japan (E-mail: sawa-p@ surg1.med.osaka-u.ac.jp).

$0022-5223 / \$ 36.00$

Copyright (c) 2013 by The American Association for Thoracic Surgery

http://dx.doi.org/10.1016/j.jtcvs.2013.02.045 cytoskeletal proteins. It has been recently suggested that cell transplantation into the IDCM heart positively modulates cellular behavior of native cardiac fibroblasts and/or coronary artery smooth muscle cells (CoASMCs), leading to upregulation of multiple cardioprotective factors in the heart. ${ }^{2}$ Inasmuch as cell transplantation is clinically limited by the cell-culture procedure and the availability of a cell processing center, cell-free therapy that enhances cardiac regeneration has long been sought in the clinical arena. $^{3}$

Prostacyclin and its analogs have been shown to upregulate expressions of various factors, such as hepatic growth factor (HGF) and vascular endothelial growth factor (VEGF) in vitro and in vivo. ${ }^{4}$ Although previously generated prostacyclin agonists are chemically unstable, being limited by the delivery method, it has recently been shown that ONO1301 is a selective prostacyclin receptor (IPR) agonist having a unique, chemically stable structure, and polymerization of ONO1301 with polylactic-co-glycolic acid copolymer (PLGA) to form a microsphere (ONO1301-MS) upregulates multiple protective factors, represented by HGF, for 3 to 4 weeks in vivo. ${ }^{5}$ We therefore 


\section{Abbreviations and Acronyms \\ CoASMC = coronary artery smooth muscle cell \\ $\mathrm{DCM}=$ dilated cardiomyopathy \\ Dd/Ds = diastolic/systolic dimensions \\ $\mathrm{EF} \quad=$ ejection fraction \\ ELISA $=$ enzyme-linked immunosorbent assay \\ GAPDH = glyceraldehyde-3-phosphate dehydrogenase \\ G-CSF = granulocyte colony stimulating factor \\ HCoASMC $=$ human coronary artery smooth muscle cell \\ HGF = hepatic growth factor \\ IPR = prostacyclin receptor \\ IDCM = idiopathic dilated cardiomyopathy \\ $\mathrm{LV}=$ left ventricular (ventricle) \\ $\mathrm{N}$ group $=$ atelocollagen sheet without ONO1301 \\ NHDF $=$ normal human dermal fibroblast \\ O group = atelocollagen sheet containing ONO1301 \\ PCR = polymerase chain reaction \\ PLGA $\quad$ polylactic-co-glycolic acid copolymer \\ $S$ group $=$ sham group \\ SDF-1 = stromal cell-derived factor- 1 \\ VEGF $\quad=$ vascular endothelial growth factor \\ vWF $\quad=$ von Willebrand factor}

hypothesized that administration of ONO1301-MS into the IDCM heart might upregulate cardiac protective factors, leading to histologic and functional reverse left ventricular (LV) remodeling.

\section{MATERIALS AND METHODS}

Experimental procedures related to animal studies were carried out under the approval of the institutional ethics committee. The investigation conformed to the "Principles of Laboratory Animal Care" formulated by the National Society for Medical Research and the "Guide for the Care and Use of Laboratory Animals" (National Institutes of Health Publication No. 85 to 23, revised 1996). All experimental procedures and evaluations were performed in a blinded manner.

\section{Cell Culture}

Normal human dermal fibroblasts (NHDFs) and human CoASMCs (HCoASMCs) were purchased from EIDIA Co, Ltd (Tokyo, Japan). The cells were cultured on 6-well plates with Dulbecco's modified Eagle's medium (Sigma-Aldrich, St Louis, Mo) supplemented with $10 \%$ fetal bovine serum (EIDIA) under 5\% carbon dioxide. Next, 1- to 1000-nmol/L ONO1301 (Ono Pharmaceutical, Osaka, Japan), dimethyl sulphoxide (Sigma-Aldrich) or dibutyryl cyclic adenosine monophosphate (Sigma-Aldrich) was added to the culture medium for 72 hours, and then the culture supernatants and cells $(\mathrm{n}=6$, respectively) were harvested and stored at $-80^{\circ} \mathrm{C}$.

\section{Procedure of ONO1301-MS Administration to the Dilated Cardiomyopathy (DCM) Hamster}

Male 20-week-old $\delta$-sarcoglycan-deficient J2N-k hamsters and J2N-n normal hamsters were purchased from Japan SLC (Shizuoka, Japan). Inasmuch as human DCM-like histopathologic features and associated functional deterioration develop in J2N-k hamsters, they have been used as an established IDCM model. ${ }^{6}$ Each hamster underwent left lateral thoracotomy under $1.5 \%$ isoflurane anesthesia $(n=66)$. Subsequently, ONO-1301 was delivered into the heart using a novel drug delivery system, in which an atelocollagen sheet (Integran sheet; Koken Co, Ltd, Tokyo, Japan) shaped "hand-drum" containing ONO1301-MS $(10 \mathrm{mg} / \mathrm{kg})$ was placed to cover the entire ventricular free wall (O group, $\mathrm{n}=22$ ). Other hamsters underwent either ONO1301-free atelocollagen sheet implantation in the same manner ( $\mathrm{N}$ group, $\mathrm{n}=21$ ) or sham operation ( $\mathrm{S}$ group, $\mathrm{n}=23$ ). After the layered closure, the hamsters were housed in a temperaturecontrolled individual cage until spontaneous or scheduled death at 2 or 4 weeks after the operation $(\mathrm{n}=5$ each).

\section{Measurement of ONO1301 Concentration in the Plasma and the Ventricular Tissue}

Under isoflurane inhalation $(5 \%)$, venous blood $(1 \mathrm{~mL})$ was sampled from the internal jugular vein, and the ventricle was then excised from the hamster at day 1 and weeks 1, 2, 4, and 8 after ONO1301 treatment $(\mathrm{n}=3$ each). The plasma was stored at $-80^{\circ} \mathrm{C}$, and the ventricle was thoroughly washed and stored at $-80^{\circ} \mathrm{C}$. The concentrations of ONO1301 in the plasma and the ventricle were measured by high-performance liquid chromatography with the tandem mass spectrometric (LC/MS/MS) detection. ${ }^{7}$

\section{Transthoracic Echocardiography}

Transthoracic echocardiography was performed using a system equipped with a 12-MHz transducer and SONOS 5500 (Agilent Technologies, Palo Alto, Calif) under isoflurane inhalation (1\%). Diastolic/systolic dimensions (Dd/Ds) and ejection fraction (EF) of the LV were measured. ${ }^{8}$

\section{Histopathology}

The heart was excised under isoflurane anesthesia (5\%) and immersionfixed with ice cold $4 \%$ paraformaldehyde. The fixed heart was embedded with either paraffin or optimal cutting temperature compound (Funakoshi, Tokyo, Japan) and transversely sliced to generate paraffin or frozen sections, respectively. The paraffin sections were stained using picrosirius red or immunohistologically labeled using anti-von Willebrand factor (vWF) antibody (DAKO, Glostrup, Denmark). Frozen sections $(7-\mu \mathrm{m}$ thick) were immunohistologically labeled using anti- $\alpha$-dystroglycan (clone: VIA4-1; Upstate Biotechnology, Lake Placid, NY), anti- $\alpha$-sarcoglycan (clone: Ad1/20A6; Novocastra, Wetzlar, Germany), anti- $\beta$-sarcoglycan (clone: bSarc/5B1; Novocastra), anti-IPR (Abcam, Cambridge, United Kingdom), or anti- $\alpha$-actin (Millipore, Billerica, Mass) antibodies. The sections were then labeled by corresponding AlexaFluor488/594conjugated secondary antibodies counterstained with 6-diamidino-2phenylindole (DAPI; Life Technologies, Calif). 3,3'-Diaminobenzidine (DAB) staining of IPR was performed using the LSAB2 kit (DAKO). Fluorescent-labeled sections were viewed under an ECLIPSE TE 200-U confocal microscope (Nikon, Tokyo, Japan). The percentage of the total area that was fibrotic, as determined by picrosirius red staining, was calculated by using a planimetric method with MetaMorph software (Molecular Device, Osaka, Japan). The number of capillaries per square millimeter was calculated by the BZ Analyzer (Keyence, Osaka, Japan) and was counted in 4 high-power fields per section (a total of 10-12 fields/heart).

\section{Real-Time Polymerase Chain Reaction}

Total RNA was isolated from the cultured cells and the free wall of the LV using the RNeasy Kit and reverse-transcribed using Omniscript Reverse 
TABLE 1. Forward and reverse primers and probe

\begin{tabular}{|c|c|c|c|}
\hline & F-primer & R-primer & Probe \\
\hline \multicolumn{4}{|l|}{ Human } \\
\hline GAPDH & GAA GGT GAA GGT CGG AGT C & GAA GAT GGT GAT GGG ATT TC & CAA GCT TCC CGT TCT CAG CC \\
\hline HGF & ATG ATG TCC ACG GAA GAG GAG A & $\begin{array}{l}\text { CAC TCG TAA TAG GCC ATC ATA } \\
\text { GTT GA }\end{array}$ & $\begin{array}{l}\text { TGC AAA CAG GTT CTC AAT GTT } \\
\text { TCC CAG C }\end{array}$ \\
\hline VEGF & $\begin{array}{l}\text { GAA GTG GTG AAG TTC ATG GAT } \\
\text { GTC T }\end{array}$ & CAC ACA GGA TGG CTT GAA GAT G & $\begin{array}{l}\text { TTC CAG GAG TAC CCT GAT GAG } \\
\text { ATC GA }\end{array}$ \\
\hline SDF-1 & CAT GCC GAT TCT TCG AAA GC & $\begin{array}{l}\text { CTA CAA TCT GAA GGG CAC AGT } \\
\text { TTG }\end{array}$ & $\begin{array}{l}\text { TGT TGC CAG AGC CAA CGT CAA } \\
\text { GCA }\end{array}$ \\
\hline G-CSF & GCT GTG GCA CAG TGC ACT CT & CCC TGG ATC TTC CTC ACT TGC TC & $\begin{array}{l}\text { CCT GCC CCA GAG CTT CCT GCT } \\
\text { CA }\end{array}$ \\
\hline \multicolumn{4}{|l|}{ Hamster } \\
\hline GAPDH & CTG CAC CAC CAC CTG CTT AGC & GCC ATG CCA GTG AGC TTC C & CTG CAC CAC CAC CTG CTT AGC \\
\hline HGF & AGG TCC CAT GGA TCA CAC AGA & GCC CTT GTC GGG ATA TCT TTC T & ACC AGC AGA CAC CAC ACC GGC A \\
\hline VEGF & GCA CTG GAC CCT GGC TTT ACT & TCA TGG GAC TTC TGC TCT CCT T & $\begin{array}{l}\text { ACC ATG CCA AGT GGT CCC AGG } \\
\text { CT }\end{array}$ \\
\hline$\alpha$-Sarcoglycan & $\begin{array}{l}\text { AAC TGA AGA GAG ACA TGG } \\
\text { CCA CC }\end{array}$ & CAG TGC TGG TCC AGG ATG AGG & $\begin{array}{l}\text { CCT CTC TCC ACC TTG CCC ATG } \\
\text { TTC A }\end{array}$ \\
\hline$\beta$-Sarcoglycan & $\begin{array}{l}\text { TCC ACT GAG AGG ATT ACC AGC } \\
\text { AAT }\end{array}$ & AGT TTG TAG CGC ACC CAG TCA C & $\begin{array}{l}\text { TCC TCA ATG GAA CTG TGA TGG } \\
\text { TCA GCC C }\end{array}$ \\
\hline$\alpha$-Dystroglycan & CAC ACA GTC ATT CCA GCT GTT GT & TCA TCC AGC TCG TCT GCA AAG & $\begin{array}{l}\text { CCT TGA GGA CCA GGC CAC CTT } \\
\text { TAT CAA }\end{array}$ \\
\hline
\end{tabular}

$\overline{G A P D H}$, Glyceraldehyde-3-phosphate dehydrogenase; $H G F$, hepatic growth factor; $V E G F$, vascular endothelial growth factor; SDF-1, stromal cell-derived factor-1; G-CSF, granulocyte colony stimulating factor.

transcriptase (Qiagen, Hilden, Germany). Quantitative polymerase chain reaction (PCR) was performed with the ABI 7500 Fast Real-Time PCR using TaqMan Universal Master Mix (Applied Biosystems, Division of Life Technologies Corporation, Carlsbad, Calif) and the designed primers/ probes (Table 1). Expression of each mRNA was normalized to that of glyceraldehyde-3-phosphate dehydrogenase (GAPDH).

\section{Western Blotting}

The $\mathrm{LV}$ free wall was homogenized and centrifuged at $1000 \mathrm{~g}$ at $4^{\circ} \mathrm{C}$ for 10 minutes to retrieve protein. Subsequently, 10 to $20 \mu \mathrm{g}$ of protein was subjected to sodium dodecyl sulfate polyacrylamide gel electrophoresis with $12.5 \%$ gels and transferred to polyvinylidene difluoride membranes (GE Healthcare, Little Chalfont, United Kingdom) using the Mini TransBlot system (170-3930; Bio-Rad Laboratories, Hercules, Calif). Protein blots in polyvinylidene difluoride membranes were incubated with each of the primary antibodies against $\alpha$-sarcoglycan or GAPDH (Abcam, Plc, Cambridge, United Kingdom). The membranes were incubated with the corresponding horseradish peroxidase-labeled secondary antibody and then visualized using the ECL system (GE Healthcare Lifesciences, Cleveland, Ohio). The band intensities were quantified by Image-J software (Wine Rasband, Bethesda, Md). The values obtained were expressed as a percentage of the value in the $\mathrm{J} 2 \mathrm{~N}-\mathrm{n}$ hamsters.

\section{Statistical Analysis}

Data are summarized as means \pm SEMs. Comparisons among groups were made with the use of 1-way analysis of variance, followed by the Tukey honestly significant difference test. Differences across the whole time course of echocardiographic data and the concentration of ONO1301 were analyzed by repeated analysis of variance including main effects of the group, time, and their interactive effects, followed by pairwise comparisons at different times using a paired $t$ test with the Bonferroni multiplicity correction. Survival curves were prepared by using the Kaplan-Meier method and compared using the log-rank test. All probability values are 2 -sided. Statistical analysis was performed by using SPSS version 11.0 (SPSS, Inc, Chicago, Ill).

\section{RESULTS}

\section{Effects of ONO1301 on the Secretion of Protective Factors in Vitro}

Dose-dependent effects of ONO1301 on each component of the cells were assessed by cultivating commercially available NHDFs, HCoASMCs in vitro. Synthesis and extracellular secretion of protective factors were quantitatively assessed by real-time PCR and enzyme-linked immunosorbent assay (ELISA; Quantikine; R\&D Systems, Minneapolis, Minn), respectively. ONO1301 treatment upregulated the expressions of HGF, VEGF, and stromal cell-derived factor-1 (SDF-1), but not granulocyte colony stimulating factor (G-CSF), in the NHDFs assessed by real-time PCR (Figure 1, $A$ ). In particular, a high concentration of ONO1301 significantly upregulated expressions of these factors. These findings were consistent with the results of ELISA for the culture supernatants (Figure 1, B). A high concentration of ONO1301 also significantly upregulated expressions of HGF, VEGF, and G-CSF, but not SDF-1, in the HCoASMCs, assessed by real-time PCR and ELISA (Figure 1, $C$ and $D$ ).

\section{Heart-Dominant Elevation of ONO1301 Concentration After ONO1301 Treatment}

The difference in the ONO1301 concentration between the plasma and the LV after ONO1301 treatment was quantitatively and serially assessed by the LC/MS/MS method. The ventricular ONO1301 concentration was markedly greater than the plasma concentration at week 1 and week 2 after the treatment, although the plasma ONO1301 concentration 
was higher than the detectable limit for this period (Figure 2, A). Both ventricular and plasma concentrations of ONO1301 were less than the detectable limit at weeks 4 and 8 .

\section{Presence of IPR in the Vasculature of the IDCM Heart}

Localization of the IPR in the heart was assessed by immunohistolabeling. IPR was present in the microvasculature component, such as vascular SMCs and endothelial cells (Figure 2, $B$ and $C$ ), but not in the cardiac fibroblasts or cardiomyocytes (Figure 2,D). Expression of the IPR was not different between the $\mathrm{J} 2 \mathrm{~N}-\mathrm{k}$ and $\mathrm{J} 2 \mathrm{~N}$-n hamsters.

\section{Preserved Cardiac Performance in the IDCM Hamster With ONO1301 Therapy}

The functional effects of ONO1301-atelocollagen sheet implantation on the IDCM heart were serially assessed by transthoracic echocardiography. LVDd/Ds and EF at 20 weeks of age, just before the treatment, were not significantly different among the 3 groups (Figure 2, E). After treatment, echocardiography showed that the LVDd/Ds and EF were significantly preserved until 4 weeks in the $\mathrm{O}$ group, compared with the $\mathrm{N}$ and $\mathrm{S}$ groups, which showed a progressive increase of LVDd/Ds and a progressive reduction of LVEF for the subsequent 8 weeks. However, even the $\mathrm{O}$ group showed progressively increased LVDd/Ds and reduced LVEF in the subsequent 4 weeks.

\section{Upregulated HGF and VEGF in the Heart After ONO1301 Treatment}

Real-time PCR was used to quantitatively assess the trend in expression of angiogenic factors, such as HGF and VEGF, in the hearts of the 3 groups and the normal hamster $(n=5$, each). Intramyocardial mRNA levels of HGF and VEGF in the $\mathrm{S}$ and the $\mathrm{N}$ groups were not significantly different from those in the normal hamster at 2 or 4 weeks (Figure 3, $A$ ). In contrast, in the $\mathrm{O}$ group, both HGF and VEGF were significantly upregulated at 2 weeks compared with the other groups. VEGF significantly upregulated in the $\mathrm{O}$ group at 4 weeks, although the HGF level in the $\mathrm{O}$ group was not significantly different from that in the other groups at 4 weeks.

\section{Increased Vasculature in the Heart After ONO1301 Treatment}

The trend in the distribution and the number of arterioles and capillaries in the heart after ONO1301 treatment was assessed by vWF-labeled sections of the 3 groups and normal hamsters ( $\mathrm{n}=5$, each). The number of vWF-positive arterioles and capillaries was significantly less in the $\mathrm{N}$ and the $S$ groups than in the normal heart at 2 and 4 weeks. In contrast, vWF-positive arterioles and capillaries were homogeneously increased in the $\mathrm{O}$ group at 2 and 4 weeks compared with the other groups (Figure $3, B ; P<.05$ ).

\section{Effect of ONO1301 on Myocardial Fibrosis}

The distribution and the quantity of interstitial collagen in the heart after ONO1301 treatment was assessed by picrosirius red-stained sections ( $n=5$, each). Interstitial collagen was significantly accumulated in the $\mathrm{J} 2 \mathrm{~N}-\mathrm{k}$ hamsters, regardless of the treatment, compared with the normal hamsters (Figure 3,C). However, collagen accumulation was significantly less in the $\mathrm{O}$ group than in the $\mathrm{S}$ and the $\mathrm{N}$ groups at 2 and 4 weeks.

\section{Reorganization of Cytoskeletal Proteins After ONO1301 Treatment}

The trend in the expression of $\alpha$-sarcoglycan in the hearts after ONO1301 treatment was comprehensively assessed by immunohistolabeling, real-time PCR, and Western blotting analysis (Figure $4, A-C$ ). $\alpha$-Sarcoglycan was homogeneously expressed around the cardiomyocytes of the normal hamster, but it was rarely expressed in the heart of the $\mathrm{N}$ or $\mathrm{S}$ groups on immunoconfocal microscopy. In contrast, $\alpha$-sarcoglycan expression was greater in the $\mathrm{O}$ group than in the $\mathrm{N}$ and $\mathrm{S}$ groups at 2 weeks, but not at 4 weeks. The mRNA of $\alpha$-sarcoglycan was significantly greater in the $\mathrm{O}$ group than in the $\mathrm{S}$ and the $\mathrm{N}$ groups at 2 weeks, but not at 4 weeks. Consistently, Western blotting analysis in the heart showed significantly upregulated $\alpha$-sarcoglycan expression at 2 weeks, but not at 4 weeks in the $\mathrm{O}$ group, compared with the $\mathrm{S}$ and the $\mathrm{N}$ groups. In addition, the trends in expressions of other cytoskeletal proteins, such as $\beta$-sarcoglycan and $\alpha$-dystroglycan, after the ONO1301 treatment were assessed by immunohistolabeling and real-time PCR (Figure $4, D$ and $E$ ). $\beta$-Sarcoglycan was rarely expressed in the J2N-k hamster heart regardless of treatment, whereas expression of $\alpha$-dystroglycan appeared to be greater in the $\mathrm{O}$ group than in the $\mathrm{S}$ and the $\mathrm{N}$ groups, although there were no significant differences.

\section{Survival Benefit of ONO1301 Treatment in IDCM}

No mortality or morbidity related to surgical procedure was identified in any of the groups. Survival of J2N-k hamsters after treatment was then assessed using the KaplanMeier method. Hamsters of the $\mathrm{N}$ and the $\mathrm{S}$ groups showed similar progression to death, primarily owing to congestive cardiac failure over the 4 weeks after treatment. In contrast, survival of hamsters in the $\mathrm{O}$ group was significantly prolonged compared with that of the other groups (Figure 5).

\section{DISCUSSION}

\section{Summary of the Findings}

In the present study, ONO1301 induced secretion of multiple cardiac protective factors such as HGF, VEGF, SDF-1, and G-CSF from NHDFs and HCoASMCs in a dosedependent manner in vitro. Epicardial implantation of an ONO1301-immersed atelocollagen sheet, which was developed as a slow-releasing drug delivery system, induced heart-dominant elevation of ONO1301 for 2 weeks in 


\section{NHDFs}

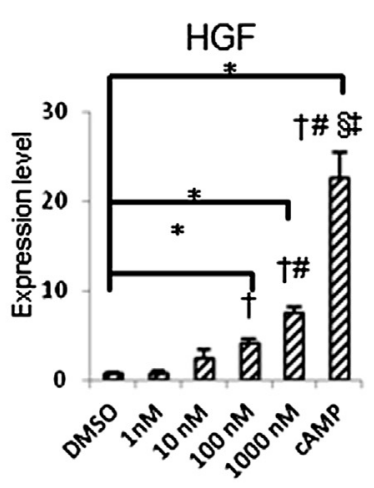

\section{HCOASMCs \\ HCOASMC}

HGF
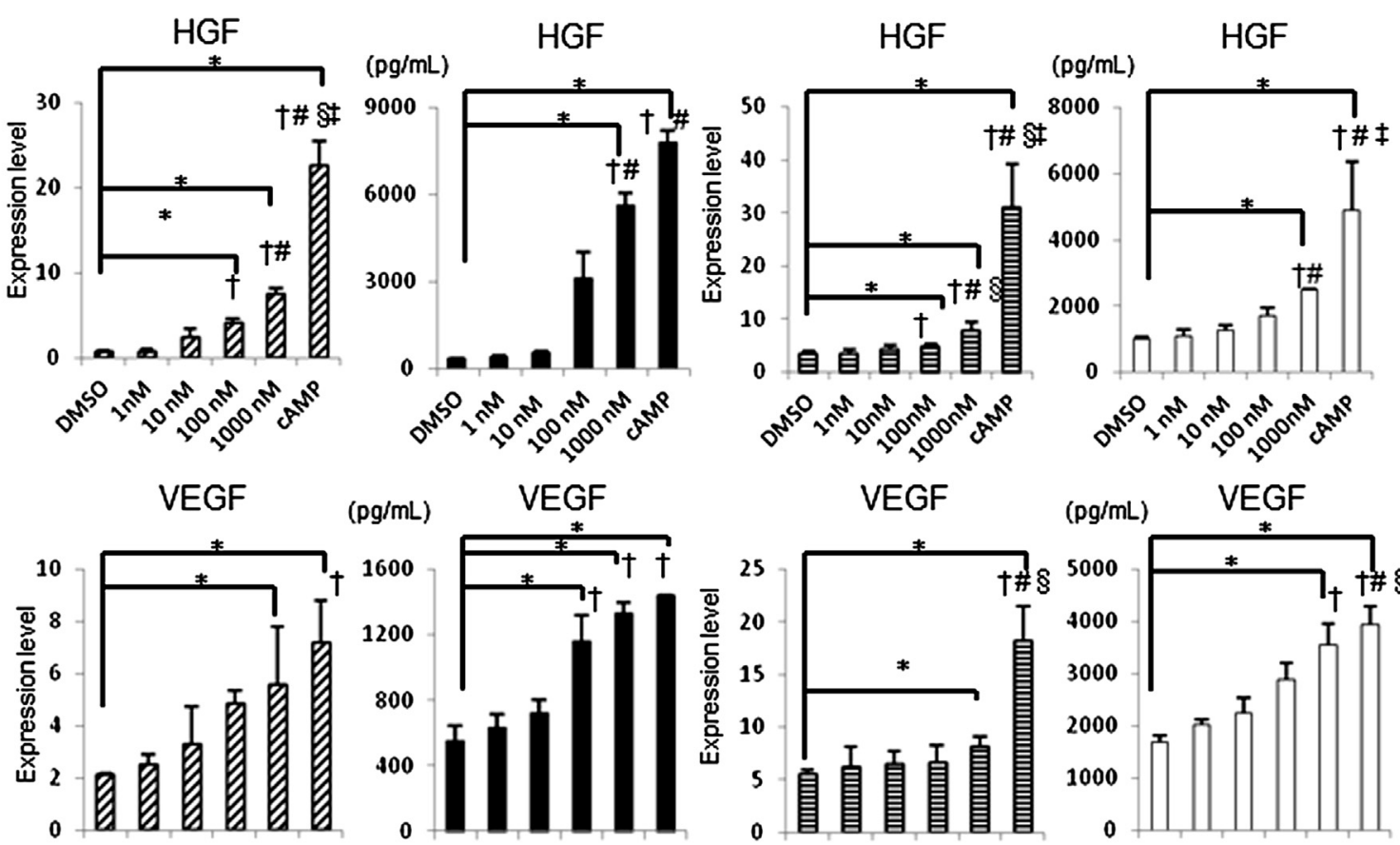

(pg/mL)

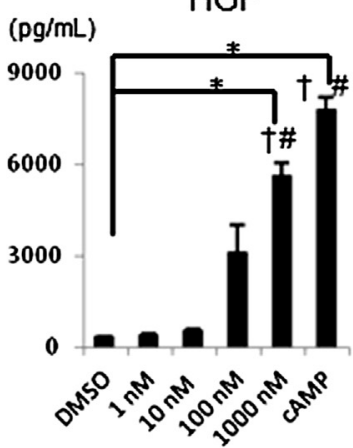

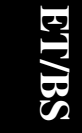
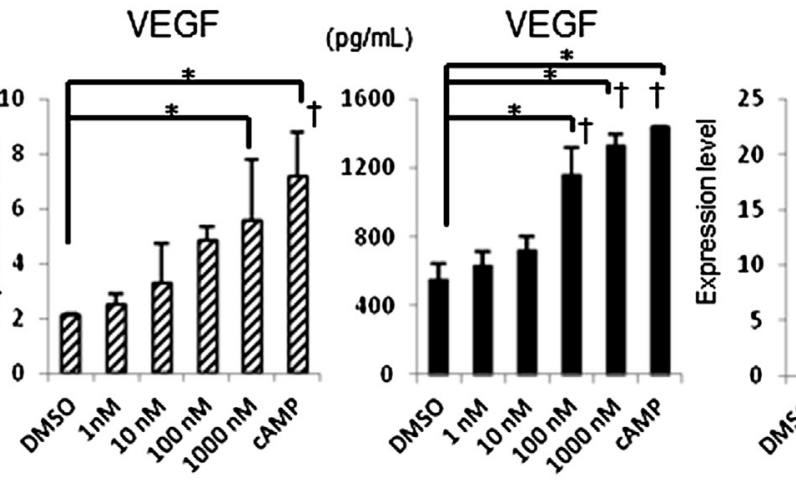

VEGF
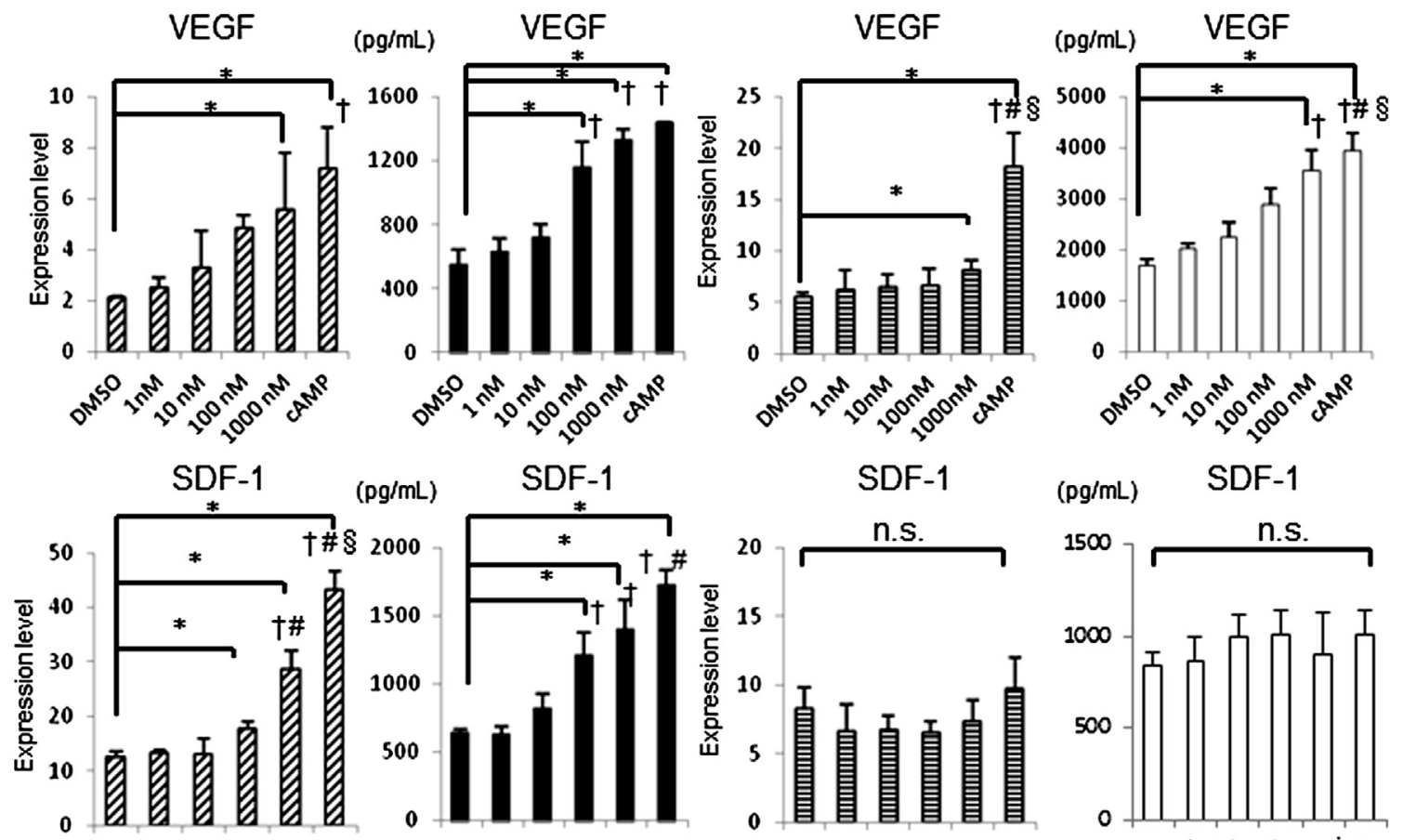

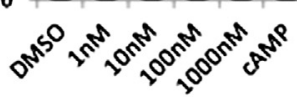
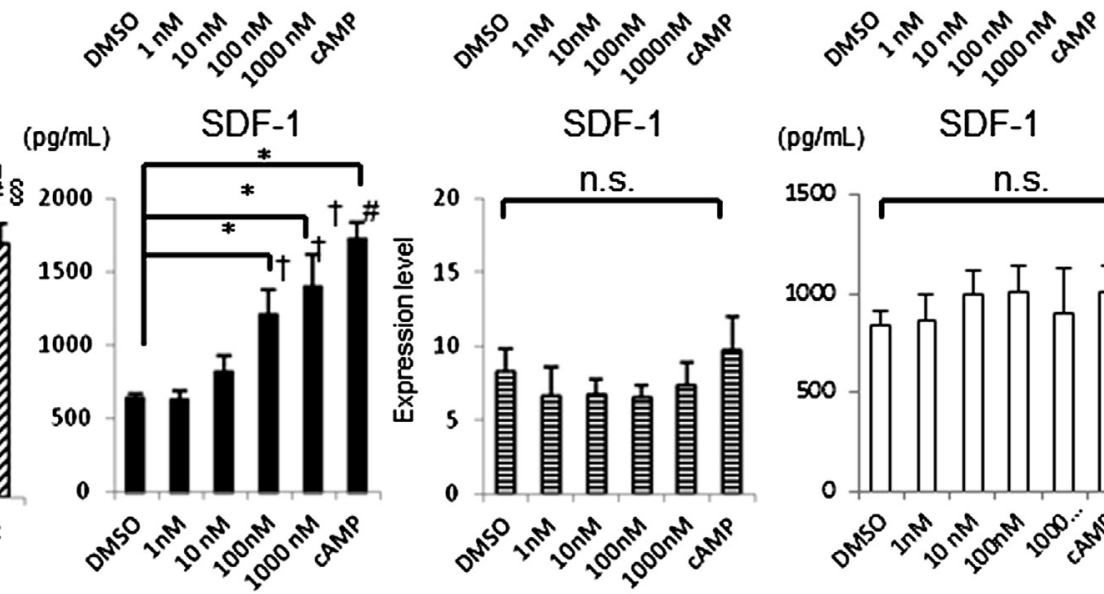

SDF-1
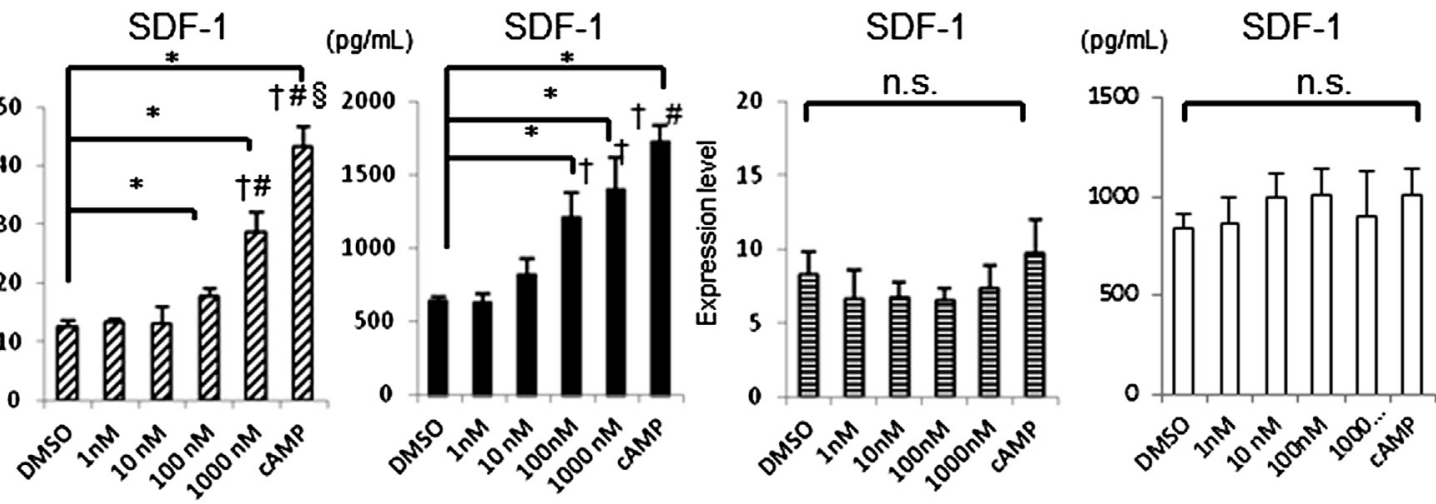

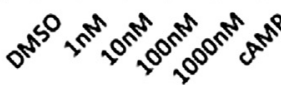
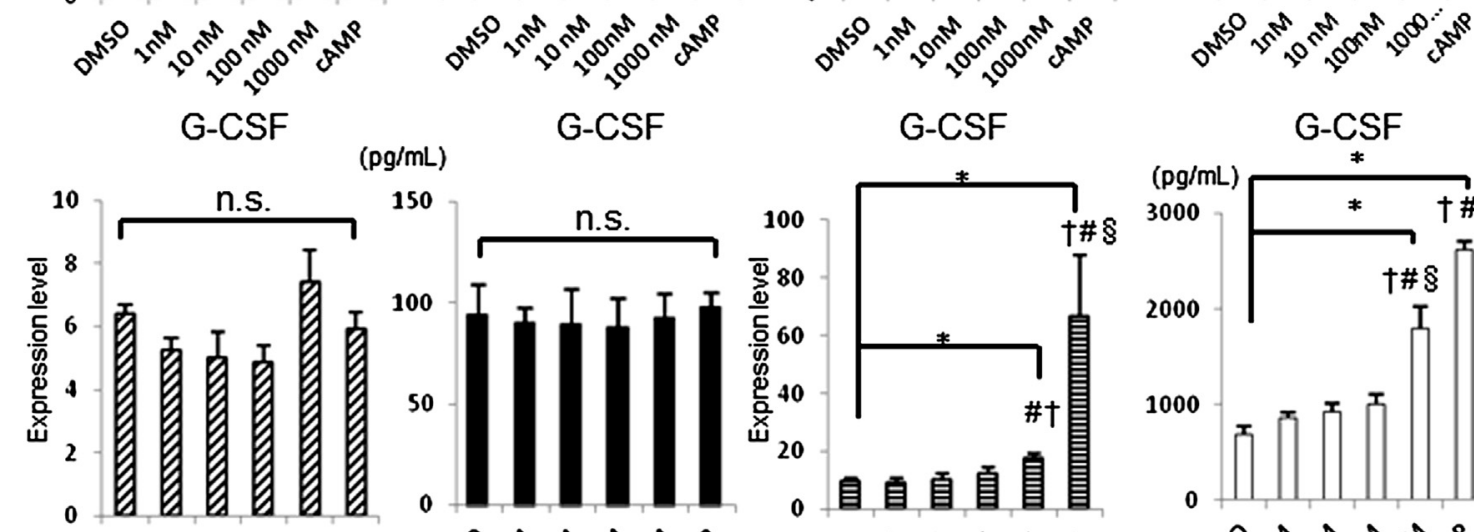

\section{(pg/mL)}

G-CSF

150
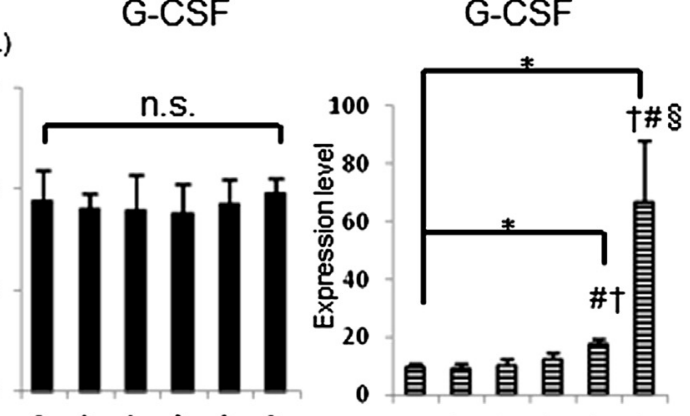

G-CSF

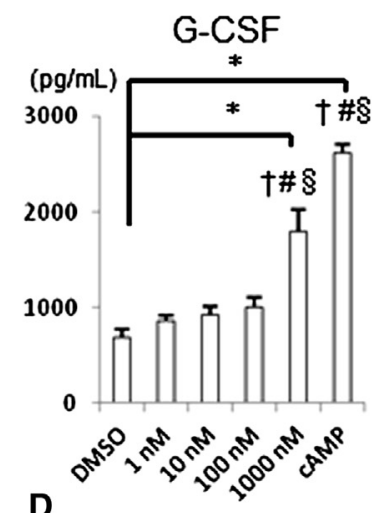

A
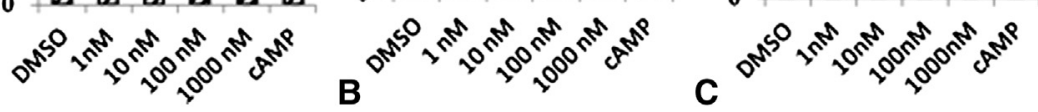

FIGURE 1. Effects of ONO1301 on the production of cardiac protective factors in NHDFs and HCoASMCs were assessed in vitro. ONO1301 treatment significantly upregulates expressions of HGF, VEGF, and SDF-1 in NHDFs, assessed by real-time PCR (A). HGF, VEGF, and SDF-1, but not G-CSF, are significantly secreted into the culture supernatant of NHDF after ONO1301 addition, measured by ELISA (B). ONO1301 addition significantly upregulates expressions of HGF, VEGF, and G-CSF, but not SDF-1, in HCoASMCs, assessed by real-time PCR and ELISA (C and D). *P <.05 versus control (DMSO); 
the $\delta$-sarcoglycan-deficient DCM hamsters. ONO1301atelocollagen sheet implantation significantly upregulated expression of HGF, VEGF, increased vasculature, attenuated fibrosis, and upregulated $\alpha$-sarcoglycan in the myocardium and, consequently, preserved cardiac performance and prolonged survival in this hamster DCM model.

\section{Rationale, Feasibility, and Safety of an Atelocollagen Sheet-Based Therapy for IDCM}

This study identified cell-dependent and dose-dependent effects of ONO1301 on the release of cardioprotective factors. The cells that were activated by ONO1301 in vitro included skin fibroblasts and CoASMCs. In addition, IPR, which is the sole receptor of ONO1301, was expressed in CoASMCs and endothelial cells, but not in cardiomyocytes or cardiac fibroblasts. These findings suggest that the target cells of ONO1301 may be the vascular SMCs and endothelial cells in the cardiac tissue. Local delivery of ONO1301 into the heart, directly targeting cardiac SMCs and endothelial cells, would thereby theoretically be useful in maximizing the therapeutic effects of ONO1301. In fact, it was shown that the ONO1301immersed atelocollagen sheet implantation therapy induced marked heart-dominant elevation of the ONO1301 level in association with significantly positive functional effects, indicating rationale and feasibility of this treatment in the IDCM heart. In addition, both the ONO1301 and the atelocollagen sheet only groups did not produce procedure-related mortality despite the deteriorated cardiac function, suggesting the safety of this treatment for IDCM heart.

There are other possible clinically relevant methods for ONO1301 delivery to treat the DCM heart, such as injection of intramyocardial microbeads, systemic intravenous/subcutaneous injection, or oral intake. However, these methods are theoretically limited by possible local damage and/or poor efficiency in the drug delivery to the cardiac tissue ${ }^{9}$ compared with the atelocollagen sheet-based drug delivery system as in this study. Intramyocardial injection of microbeads may also prove to be more efficacious, but further studies will be required to establish the optimal delivery methods of ONO1301 into the heart in preclinical and subsequent clinical studies.

\section{Therapeutic Effects and Underlying Mechanisms of ONO1301 Sheet Therapy}

Therapeutic efficiency of this treatment on this $\delta$-sarcoglycan-deficient hamster IDCM model was assured in this study by comparing with the 2 control groups, in which the sham operation or placement of atelocollagen sheet only was performed. Global systolic cardiac function, assessed by echocardiography, was significantly preserved in the ONO1301-treated hamsters compared with the other groups, and most important, the life expectancy of the J2N-k hamsters was prolonged by this treatment. These important positive findings would be explained by multiple fundamental effects of this treatment, including increased myocardial blood flow, reduction of myocardial fibrosis, and reorganization of cytoskeletal proteins.

It has been reported that ONO1301 acts as an inducer of multiple cardioprotective factors in ischemic cardiac diseases. ${ }^{5}$ Effects of ONO1301 on the IDCM heart, however, are poorly understood. Although the clinical manifestations of end-stage IDCM are similar to those of end-stage ischemic cardiomyopathy, typical IDCM is characterized by a decreased vascular network, increased fibrous components, and decreased expression of cytoskeletal proteins in a global and homogeneous manner. ${ }^{10,11}$ This study also identified multiple endogenous factors upregulated by the ONO1301atelocollagen sheet, such as HGF, a unique growth factor with antifibrosis and angiogenesis effects, ${ }^{8,12}$ or VEGF, an important mediator of angiogenesis. ${ }^{4}$ In addition, SDF-1 or G-CSF by the ONO1301 treatment in this study may have contributed to therapeutic stem cell homing and activation. ${ }^{13,14}$ Further studies will be required to determine whether these agents induce regenerative responses.

The potential effects of these endogenous factors were well correlated with the pathologic changes in this study, such as increased vasculature, attenuated fibrosis, or upregulated $\alpha$-sarcoglycan. Of them, increased blood flow may be one of the major mechanisms responsible for the positive therapeutic effects of the ONO1301 in this study. It has been shown that prostacyclin and prostacyclin-inducing HGF/VEGF bring a multiplier effect of vasodilation and new vessel formation responsible to regional ischemic insult. ${ }^{15,16}$ In addition, genetic deletion of IPR had an important role on progression of cardiovascular disease. ${ }^{15}$

It is also interesting that cytoskeletal proteins were remodeled by the ONO1301 treatment in this study. Immunohistolabeling in this study demonstrated the transient reexpression of $\alpha$-sarcoglycan and $\alpha$-dystroglycan in the $\mathrm{O}$ group. It was speculated that $\alpha$-sarcoglycan can be recycled from the plasma membrane differently from other sarcoglycans, ${ }^{17}$ and inhibition of Smad3 associated with transforming growth factor $\beta$ signal pathway suppressed by prostacyclin or HGF, brings to $\alpha$-sarcoglycan gene expression. ${ }^{18}$

\footnotetext{
$\dagger P<.05$ versus ONO1301 $(1 \mathrm{nmol} / \mathrm{L}) ; \# P<.05$ versus ONO1301 $(10 \mathrm{nmol} / \mathrm{L}) ; \oint P<.05$ versus ONO1301 $(100 \mathrm{nmol} / \mathrm{L}) ; \ddagger P<.05$ versus ONO1301 $(1000$ nmol/L). NHDF, Normal human dermal fibroblast; HCoASMC, human coronary artery smooth muscle cell; $H G F$, hepatic growth factor; $V E G F$, vascular endothelial growth factor; $S D F-1$, stromal cell-derived factor-1; $P C R$, polymerase chain reaction; $G$-CSF, granulocyte colony stimulating factor; $E L I S A$, enzyme-linked immunosorbent assay; $D M S O$, dimethyl sulphoxide; $c A M P$, cyclic aminophosphatase; n.s., not significant.
} 

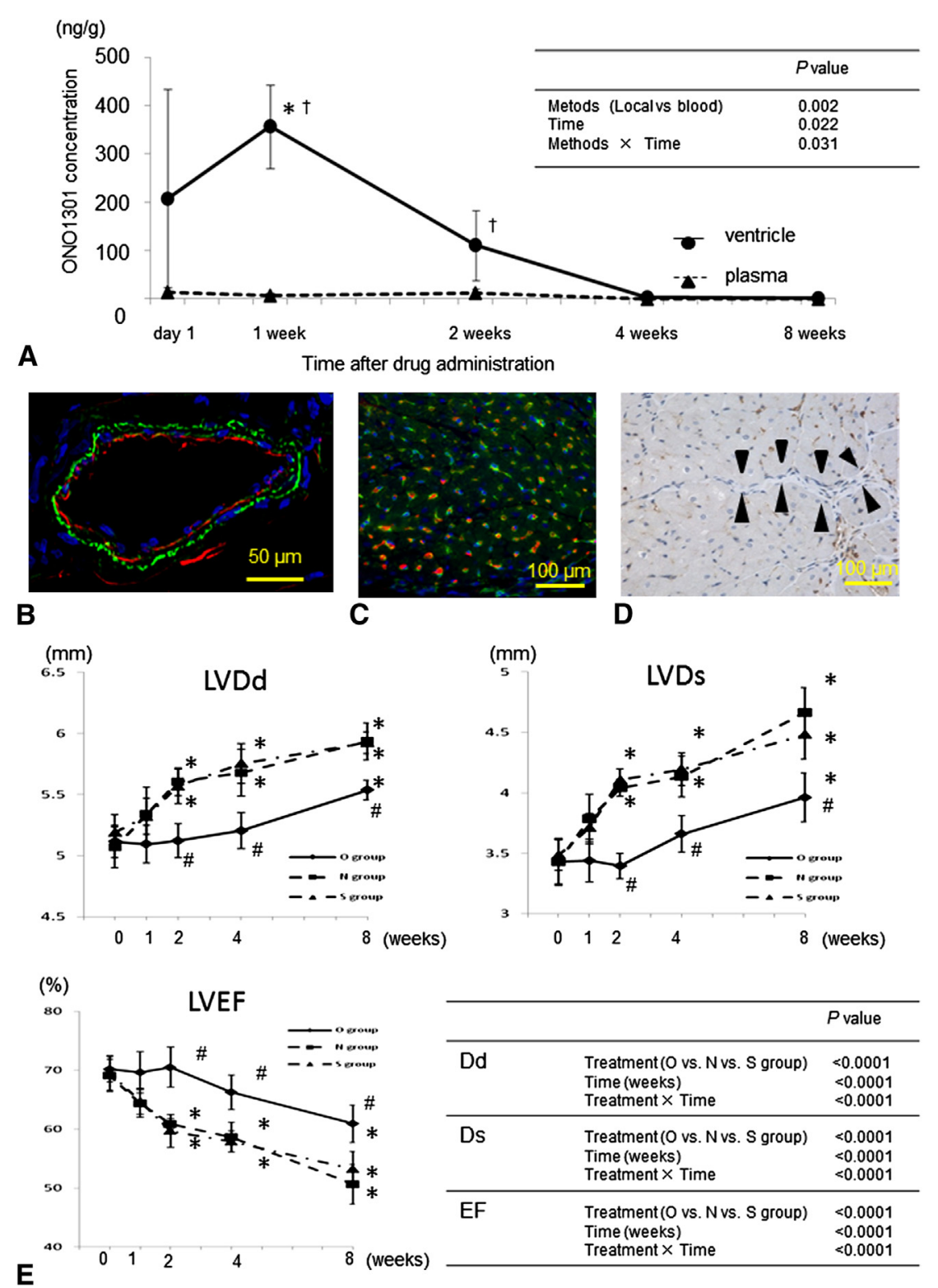

FIGURE 2. Levels of ONO1301 in cardiac tissue and plasma were serially quantified after implantation of ONO1301-eluted atelocollagen sheet for the DCM heart (A). ONO1301 is detected in both samples for 2 weeks after treatment, while the level of ONO1301 in the ventricle is significantly and markedly higher than in the plasma at weeks 1 and 2 after the treatment. $* P<.05$ versus 8 weeks; $\nmid P<.05$ versus plasma concentration Immunofluorescence staining for IPR and alphaactin in the DCM heart shows that IPR is positive in the vascular smooth muscle cells and the endotherial cells (B and C). Green, Filamentous-actin; red, IPR; blue, nuclei. 3,3'-diaminobenzidine staining, which produces a brown color, shows that IPR is expressed in the microvasculature, but not in cardiac fibroblasts and cardiomyocytes (arrowhead, cardiac fibroblast) (D). Changes in LVDd/Ds, and LVEF after treatment were serially measured by transthoracic echocardiography (E). These 4 parameters of the LV are preserved until 4 weeks after ONO1301 treatment compared with the other groups. However, ONO1301 treatment does not arrest the progression in dilatation of the dimensions and deterioration of the $\mathrm{EF}$ in the subsequent 4 weeks. $\# P<.05$ versus $\mathrm{N}$ and $\mathrm{S}$ group; $* P<.05$ versus 0 weeks. $D C M$, Dilated cardiomyopathy; $L V$, left ventricular (ventricle); $D d / D s$, diastolic/systolic dimensions; $E F$, ejection fraction; $I P R$, prostacyclin receptor.

Moreover, regarding the transient reexpression of $\alpha$-dystroglycan, Kondoh and associates ${ }^{9}$ suggested that the reconstruction of $\alpha$-dystroglycan may occur because the sarcoglycan might mask the matrix metalloproteinase cleavage site on dystroglycan and/or matrix metalloproteinase activity might be inhibited by HGF. In addition, $\beta$-sarcoglycan was rarely expressed after the ONO1301 treatment in our study. Kawada and colleagues ${ }^{19}$ reported that both $\beta$ - and $\delta$-sarcoglycan were completely missing, but $\alpha$ - and $\gamma$-sarcoglycan were weakly expressed in the J2N-k hamster, and transfer of the $\delta$-sarcoglycan gene could express not only $\delta$ - but the other 3 sarcoglycans. These findings might suggest the limitation of this drug therapy for reorganization of cytoskeletal proteins, but Hack and coworkers ${ }^{20}$ reported that the 

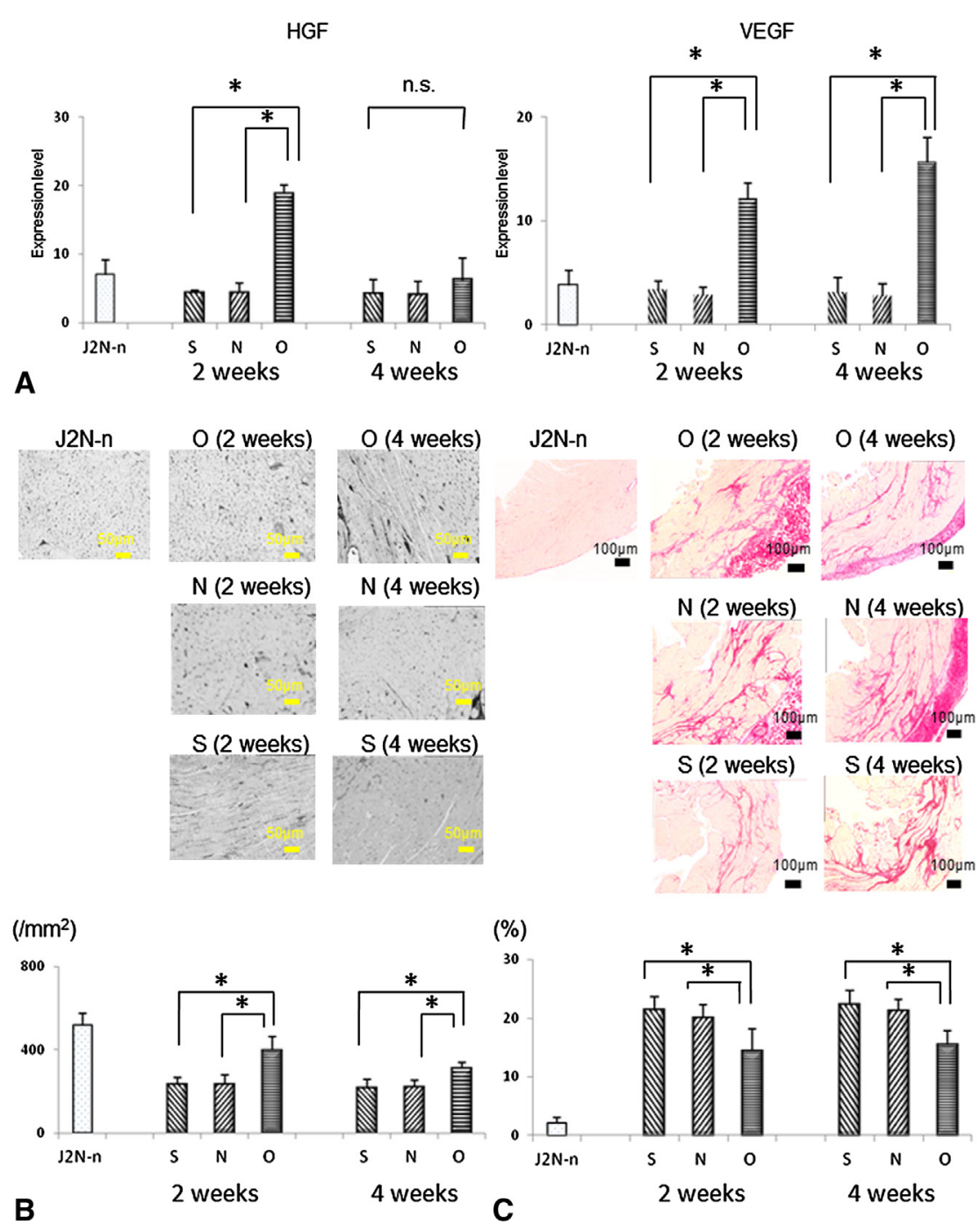

FIGURE 3. Expressions of HGF and VEGF in the cardiac tissue were assessed by real-time PCR, which shows greater expression of the 2 factors in the O group at 2 and 4 weeks compared with the other groups ( $\mathrm{n}=5$ for each group) (A). Capillary density in the hearts was assessed by immunohistolabeling for vWF, which shows a greater number of capillaries in the ONO1301-treated hearts than in the other groups at 2 and 4 weeks ( $\mathrm{n}=5$ for each group) (B). Interstitial fibrosis in the heart was assessed by picrosirius red staining, which shows less accumulation of fibrosis in the ONO1301-treated hearts than in the other groups at 2 and 4 weeks ( $\mathrm{n}=5$ for each group) (C). ${ }^{*} P<.05$ versus $\mathrm{O}$ group. $H G F$, Hepatic growth factor; $V E G F$, vascular endothelial growth factor; $P C R$, polymerase chain reaction. $v W F$, von Willebrand factor.

expression of sarcoglycans, even in small amounts, prevented the damage of cardiomyocyte. Reorganization of $\alpha$ sarcoglycan by the ONO1301 therapy might thus contribute to preserve cardiac function.

Although the level of prostacyclin in the heart in response to the ONO1301 treatment was not investigated in this study, it may be paradoxically elevated by the thromboxane synthase inhibitory activity of the ONO1301 on the heart, ${ }^{21}$ possibly producing synergetic positive effects on the IDCM heart. In addition, it is interesting to research the involvement of the neurohormonal activations in the heart, such as adrenergic system, plasma renin activity, or endothelin, by the ONO1301 treatment. ${ }^{22}$

\section{Clinical Perspectives}

The atelocollagen sheet-based local ONO1301 delivery therapy globally reversed reduced vascular density, increased fibrosis, and reduced cytoskeletal proteins in the myocardium, all of which were the typical pathologic features in the human IDCM heart, ${ }^{10,11}$ suggesting potential therapeutic benefits of this treatment for IDCM in the clinical scenario. In addition, safety of this treatment shown in this study warrants further preclinical study, including dose-response relation to explore minimum and maximal effective dose of the ONO1301 in the "GLP" standard. A very narrow dose necessary to achieve a positive response may prohibit this agent from clinical trials. 


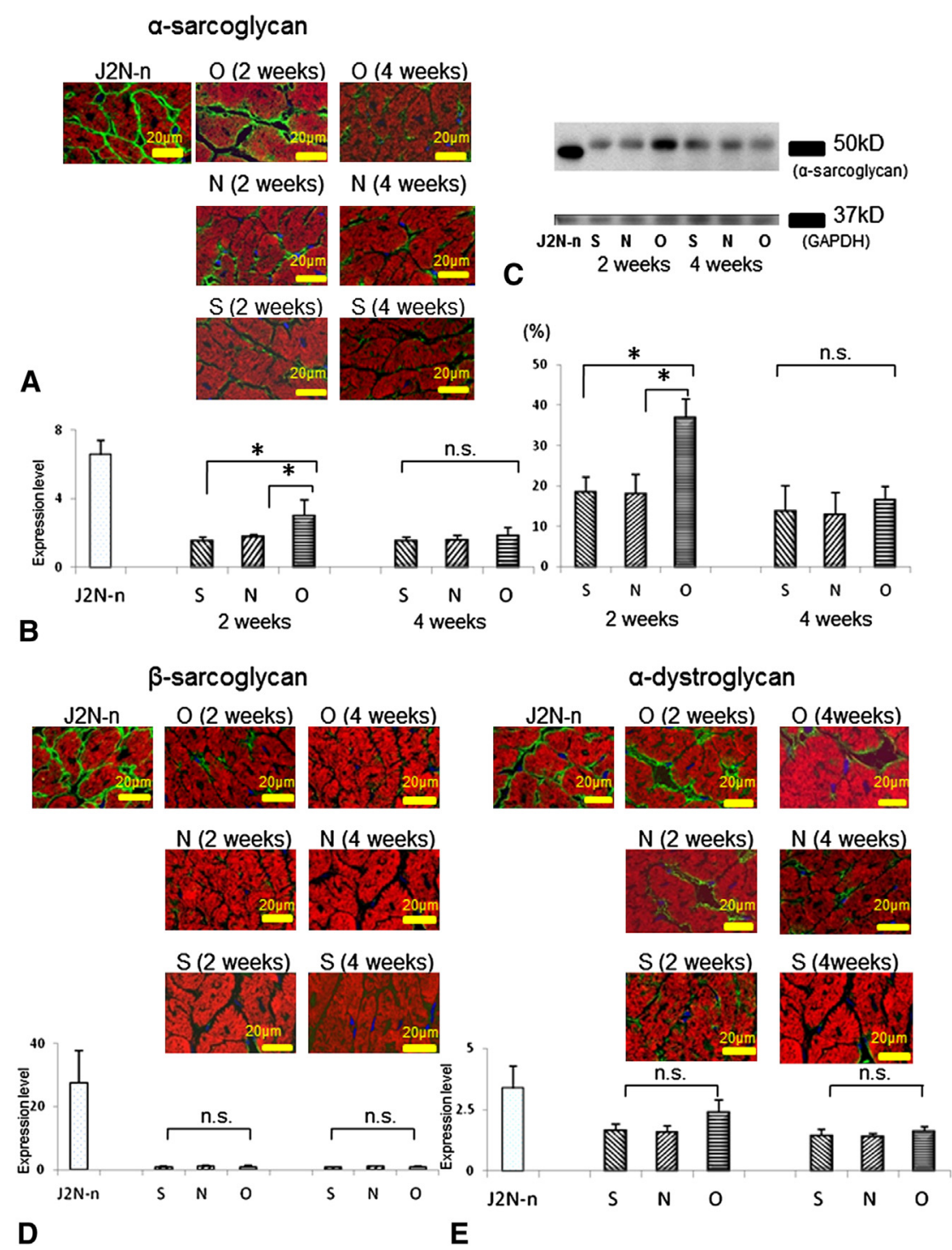

FIGURE 4. Expression of $\alpha$-sarcoglycan, $\beta$-sarcoglycan, and $\alpha$-dystoglycan in the heart after ONO1301 treatment was assessed by immunohistolabeling, real-time PCR, and Western blot analysis. Immunohistolabeling shows that $\alpha$-sarcoglycan is clearly expressed around the cardiomyocytes in the normal hamsters, but not in the $\mathrm{N}$ or S group (A). Of note, $\alpha$-sarcoglycan is expressed in the O group at 2 weeks but not at 4 weeks. Green, $\alpha$-Sarcoglycan; red, filamentous-actin; blue, nuclei. Quantitative real-time PCR shows a significantly greater expression of $\alpha$-sarcoglycan in the $\mathrm{O}$ group than in the $\mathrm{N}$ or $\mathrm{S}$ group at 2 weeks, but not at 4 weeks ( $\mathrm{n}=5$ for each group at each time point, $* P<.05$ vs $\mathrm{O}$ group) (B). Consistently, Western blot analysis and the quantitative results of band intensities, which are expressed as a percentage of the value of the J2N-n hamsters, show significantly greater expression of $\alpha$-sarcoglycan in the $\mathrm{O}$ group than in the $\mathrm{N}$ or $\mathrm{S}$ group at 2 weeks, but not at 4 weeks $(\mathrm{n}=5$ for each group at each time point, $* P<.05 \mathrm{vs} \mathrm{O}$ group) (C). Expression of $\beta$-sarcoglycan in the DCM hamsters is not detected even after ONO1301 treatment (D). Green, $\beta$-sarcoglycan; red, filamentous-actin; blue, nuclei. $\alpha$-Dystroglycan is rarely expressed in the $\mathrm{N}$ or $\mathrm{S}$ group, although its expression is upregulated in the $\mathrm{O}$ group (E). There are no significant differences between the $\mathrm{O}$ group and the other groups at 2 weeks. Green, $\alpha$-Dystroglycan; red, filamentous-actin; blue, nuclei. PCR, Polymerase chain reaction; $n$.s., not significant.

Re-treatment of epicardial implantation of the sheet containing ONO1301 might be technically challenging; however, technical modulation of microsphere generation, such as gelatin hydrogel, might induce further developments to generate a longer-release drug-delivery system than the method used in the present study. ${ }^{23}$

\section{Study Limitations}

This study was limited by use of a transgenic rodent model. The $\delta$-sarcoglycan-deficient IDCM model used in this study is not completely relevant to human IDCM that shows a number of etiologic and pathologic variations. However, positive functional and pathologic effects 


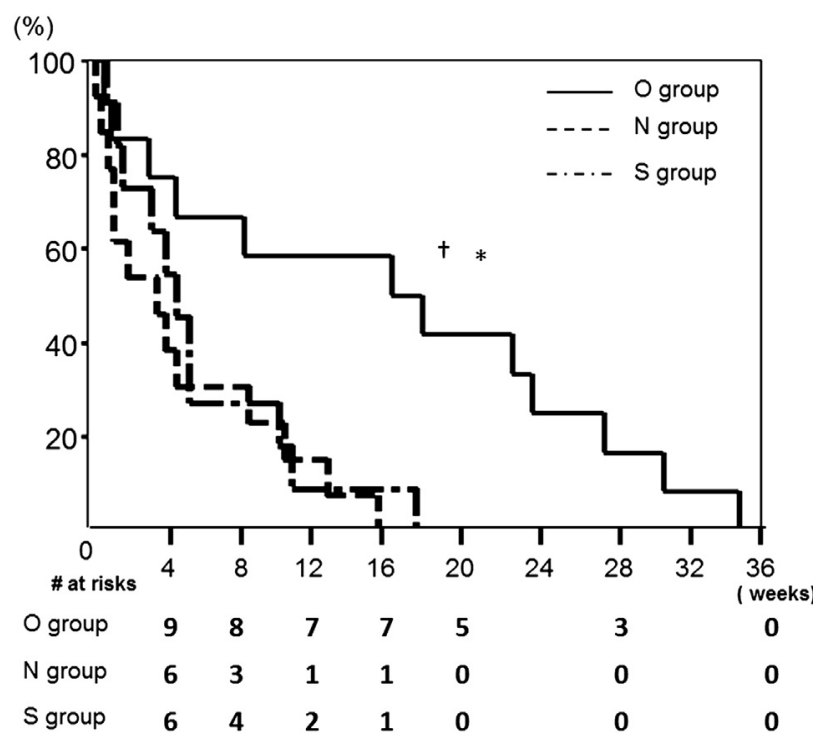

FIGURE 5. Survival after treatment was assessed by the Kaplan-Meier method. There is no significant difference between the $\mathrm{N}(\mathrm{n}=11)$ and $\mathrm{S}$ groups $(\mathrm{n}=13)$, whereas the $\mathrm{O}$ group $(\mathrm{n}=12)$ shows a significantly greater survival than the other groups ( ${ }^{*} P<.05$ vs $\mathrm{S}$ group; $\dagger P<.05$ vs $\mathrm{N}$ group).

associated with upregulated protective factors would be sufficient to prove the principal concept of this treatment. Agents that are beneficial in these mutant hamsters may not be beneficial in humans because the mechanisms responsible for the beneficial effects may be different in humans. However, further pathologic and functional studies for human DCM heart samples, in comparison with the deficient hamster, may be useful to strengthen the findings of this study.

Poor availability of the antibodies and genome sequences in the hamster limited in-depth evaluation of the mechanisms responsible for this treatment, which is warranted to be supplemented by murine IDCM model. ${ }^{24}$

\section{CONCLUSIONS}

ONO1301 reorganized cytoskeletal proteins, especially $\alpha$-sarcoglycan, increased capillaries, and reduced fibrosis through the upregulation of cardiac protective factors, leading to functional recovery and prolonged survival in the $\delta$-sarcoglycan-deficient IDCM hamster. A preclinical study to explore the optimal, clinically relevant protocol is warranted.

We thank Masako Yokoyama, Akima Harada, and Motoko Shiozaki for their excellent technical assistance.

\section{References}

1. Rajnoch C, Chachques JC, Berrebi A, Bruneval P, Benoit MO, Carpentier A. Cellular therapy reverses myocardial dysfunction. J Thorac Cardiovasc Surg. 2001; 121:871-8.

2. Nakamura T, Matsumoto K, Mizuno S, Sawa Y, Matsuda H, Nakamura T. Hepatocyte growth factor prevents tissue fibrosis, remodeling, and dysfunction in cardiomyopathic hamster hearts. Am J Physiol Heart Circ Physiol. 2005;288:H2131-9.
3. Juan CC. Cellular cardiac regenerative therapy in which patients? Expert Rev Cardiovasc Ther. 2009;7:911-9.

4. Matsumoto K, Okazaki H, Nakamura T. Novel function of prostaglandins as inducers of gene expression of HGF and putative mediator of tissue regeneration. J Biochem. 1995;117:458-64.

5. Iwata H, Nakamura K, Sumi M, Ninomiya M, Sakai Y, Sata M, et al. Local delivery of synthetic prostacycline agonist augments collateral growth and improves cardiac function in a swine chronic cardiac ischemia model. Life Sci. 2009;85:255-61

6. Mitsuhashi S, Saito N, Watano K, Igarashi K, Tagami S, Kikuchi K, et al. Defect of Delta-sarcoglycan gene is responsible for development of dilated cardiomyopathy of a novel hamster strain, J2N-k: calcineurin/PP2B activity in the heart of J2N-k hamster. J Biochem. 2003;134:269-76.

7. Saini SG, Wani AT, Vashney B, Ahmed T, Rajan SK, Paliwal LJ. Validation of the LC-MS/MS method for the quantification of mevalonic acid in human plasma and determination of the matrix effect. J Lipid Res. 2006;47:2340-5.

8. Miyagawa S, Sawa Y, Taketani S, Kawaguchi N, Nakamura T, Matsuda H, et al. Myocardial regeneration therapy for heart failure: hepatocyte growth factor enhances the effect of cellular cardiomyoplasty. Circulation. 2002; 105:2556-61.

9. Kondoh H, Sawa Y, Miyagawa S, Matsumiya S, Sakakida-Kitagawa S, Matsuda $\mathrm{H}$, et al. Longer preservation of cardiac performance by sheet-shaped myoblast implantation in dilated cardiomyopathic hamsters. Cardiovasc Res. 2006;69:466-75

10. Neglia D, Michelassi C, Trivieri MG, Sambuceti G, Giorgetti A, Parodi O, et al Prognostic role of myocardial blood flow impairment in idiopathic left ventricular dysfunction. Circulation. 2002;105:186-93.

11. Towbin JA. The role of cytoskeletal proteins in cardiomyopathies. Curr Opin Cell Biol. 1998;10:131-9.

12. Taniyama Y, Morishita R, Aoki M, Hirooka K, Yamasaki K, Ogihara T, et al. Angiogenesis and antifibrotic action by hepatocyte growth factor in cardiomyopathy. Hypertension. 2002;40:47-53.

13. Saxena A, Fish JE, White MD, Yu S, Smyth JW, Srivastava D, et al. Stromal cellderived factor- $1 \alpha$ is cardioprotective after myocardial infarction. Circulation. 2008; 117:2224-31

14. Stanford SJ, Pepper JR, Mitchell JA. Release of GM-CSF and G-CSF by human arterial and venous smooth muscle cell: differential regulation by COX-2. $\mathrm{Br}$ J Pharmacol. 2000;129:835-8.

15. Fetalvero KM, Martin KA, Hwa J. Cardioprotective prostacyclin signaling in vascular smooth muscle cell. Prostaglandins Other Lipid Mediat. 2007;82: 109-18.

16. Hiraoka K, Koike H, Yamamoto S, Tomita N, Yokoyama C, Morishita R, et al. Enhanced therapeutic angiogenesis by cotransfection of prostacyclin synthase gene or optimization of intramuscular injection of naked plasmid DNA. Circula tion. 2003;108:2689-96.

17. Draviam RA, Wang B, Shand SH, Xiao X, Watkins SC. Alpha-sarcoglycan is recycled from the plasma membrane in the absence of sarcoglycan complex assembly. Traffic. 2006; 7:793-810.

18. Hernández-Hernández JM, Delgado-Olguín P, Aguillón-Huerta V, FurlanMagaril M, Recillas-Targa F, Coral-Vázquez RM. Sox9 represses alphasarcoglycan gene expression in early myogenic differentiation. $J \mathrm{Mol} \mathrm{Biol}$. 2009;394:1-14.

19. Kawada T, Nakaturu Y, Sakamoto A, Koizumi T, Shin WS, Toyooka T, et al Strain- and age-dependent loss of sarcoglycan complex in cardiomyopathic hamster hearts and its re-expression by delta-sarcoglycan gene transfer in vivo. FEBS Lett. 1999;458:405-8.

20. Hack AA, Lam MY, Cordier L, Shoturma DI, Ly CT, Hadhazy MA. Differential requirement for individual sarcoglycans and dystrophin in the assembly and function of the dystrophin-glycoplotein complex. J Cell Sci. 2000 113:2535-44.

21. Yamanaka S, Miura K, Yukimura T, Okumura M, Yamamoto K. Putative mechanism of hypotensive action of platelet-activating factor in dogs. Circ Res. 1992; 70:893-901

22. Somova LI, Mufunda JJ. Renin-angiotensin-aldosterone system and thromboxane A2/prostacyclin in normotensive and thromboxane A2/prostacyclin in normotensive and hypertensive black Zimbabweans. Ethn Dis. 1992;2:27-34.

23. Takaoka R, Hikasa Y, Hayashi K, Tabata Y. Bone regeneration by lactoferrin released from a gelation hydrogel. J Biomater Sci Polym Ed. 2010;22:1581-9.

24. Lu D, Ma Y, Zhang W, Bao D, Dong W, Zhang L, et al. Knockdown of cytochrome P450 2E1 inhibits oxidative stress and apoptosis in the cTnT (R141W) dilated cardiomyopathy transgenic mice. Hypertension. 2012;60:81-9. 